\title{
Robust Weyl points in a 1D superlattice with transverse spin-orbit coupling
}

\author{
Xi-Wang Luo and Chuanwei Zhang* \\ Department of Physics, The University of Texas at Dallas, Richardson, Texas 75080-3021, USA
}

\begin{abstract}
Weyl points, synthetic magnetic monopoles in the 3D momentum space, are the key features of topological Weyl semimetals. The observation of Weyl points in ultracold atomic gases usually relies on the realization of high-dimensional spin-orbit coupling (SOC) for two pseudospin states (i.e., spin-1/2), which requires complex laser configurations and precise control of laser parameters, thus has not been realized in experiment. Here we propose that robust Wely points can be realized using 1D triple-well superlattices (spin-1/three-band systems) with 2D transverse SOC achieved by Raman-assisted tunnelings. The presence of the third band is responsible to the robustness of the Weyl points against system parameters (e.g., Raman laser polarization, phase, incident angle, etc.). Different from a spin-1/2 system, the non-trivial topology of Weyl points in such spin-1 system is characterized by both spin vector and tensor textures, which can be probed using momentumresolved Rabi spectroscopy. Our proposal provides a simple yet powerful platform for exploring Weyl physics and related high-dimensional topological phenomena using high pseudospin ultracold atoms.
\end{abstract}

\section{INTRODUCTION}

Weyl semimetal, an exotic topological phase of matter, possesses novel quasi-particle excitations behaving as Weyl fermions in the bulk and intriguing Fermi arcs on the surface [1-5]. The key feature of Weyl semimetal is the appearance of Weyl points (gapless points in the band structure with linear dispersion in 3D momentum space) characterized by nontrivial topological invariants $[3,4]$. Weyl point does not depend on symmetry except the translational symmetry of the crystal lattice, and is the most robust degeneracy which can only be gapped out when annihilates with another Weyl point with opposite topological charge. Weyl fermions may exhibit non-trivial electromagnetic responses to external gauge field [6-8]. Due to the fundamental importance of Weyl fermions and the potential application of surface states, significant theoretical and experimental progresses have been made for exploring Weyl physics in both solid-state materials [9-18] and synthetic systems such as ultracold atomic gases [19-26], photonic [27-32] and acoustic crystals [33]. In contrast to solid-state materials whose complicated band structures make the probing of Weyl-fermion topology elusive, synthetic systems are simple, clean and highly controllable. In particular, recent experimental realization of $1 \mathrm{D}$ and $2 \mathrm{D}$ spin-orbit coupling and synthetic gauge field in ultracold atoms makes the atomic system one of the most promising platforms for studying topological effects and novel state of matter [34-47].

So far most ultracold atom based schemes [19-26] for realizing Weyl physics rely on the generation of 3D spinorbit coupling for two pseudospin states (i.e., spin-1/2) in either optical lattices or free space, which require complex laser setups. Furthermore, such Weyl points are usually very sensitive to laser parameters (e.g., phases, polarizations and incident angles), making the experimental realization very challenging with current tech- nique. Weyl points were also proposed in quasi-particle spectra of BCS superfluids with spin-orbit coupling [4850], but the experimental realization of such superfluid is difficult due to heating. Finally, probing non-trivial topology of Weyl points for spin-1/2 systems is another challenging task, which requires measurements in various spin bases [51] where many precisely controlled pulses are needed.

In this paper, we propose a much simpler scheme to realize robust Weyl points and probe their non-trivial topology using a 1D superlattice. Instead of a spin- $1 / 2$ system, we consider a three-band (i.e., spin-1) model using a triple-well superlattice, with neighbor site tunnelings assisted by three Raman lasers. The Raman-assisted tunnelings also induce momentum transfer on the transverse plane, leading to $2 \mathrm{D} \mathrm{SOC}$ in the transverse free space. Our main results are:

i) The three-band system supports two Weyl points, corresponding to the degeneracy between two-lower and two-upper bands, respectively. Therefore they cannot annihilate with each other and any change in system parameters only shifts their positions, leading to the robustness against variations of laser parameters (e.g., incident angle, intensity, phase, detuning and polarization). Such robustness originates from the higher dimensional Hilbert space enabled by the spin-1 system, which reduces the requirement for precisely controlled SOC for spin- $1 / 2$ systems.

ii) For any two neighbor bands, the corresponding surface states would connect the Weyl point to infinite momentum, indicating that there is a virtual Weyl point (with opposite charge) at infinity. This can also be seen by the trajectory of the Weyl point, which may annihilate with its virtual partner only when it is shifted to infinity for certain critical system parameters. Away from these critical values, the Weyl points persist.

iii) Though the Berry flux around the Weyl point possesses monopole behavior, the spin textures for such spin- 


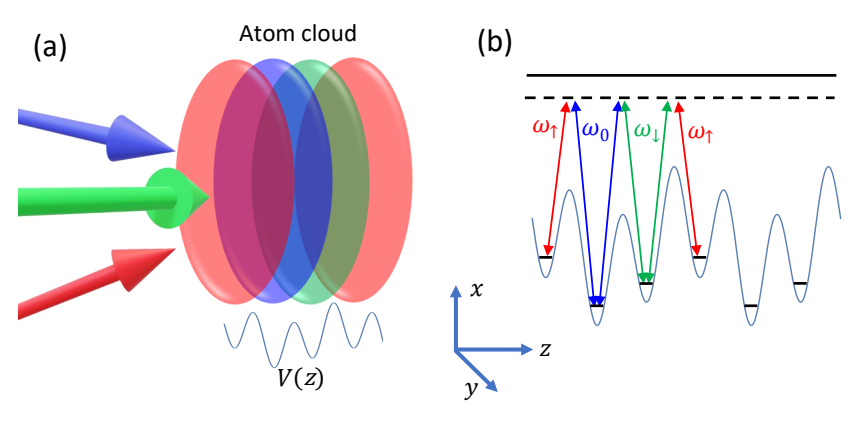

FIG. 1: (a) Experimental scheme for generating robust Weyl points using a triple-well superlattice. Three Raman lasers are used to induce the neighbor site tunnelings. (b) Corresponding level structure and two-photon Raman transitions in the superlattice.

1 system is very different from the spin- $1 / 2$ system [2326]. Since the spin-1 vector may go into the Bloch sphere representing the phase space and even vanish by crossing the center, the non-trivial topology of the Weyl point is characterized not only by the spin vectors, but also the spin tensors. We also find that there is a one-to-one correspondence between the non-trivial Chern number and the number of vanishing points in the spin vector textures around the Weyl point.

$i v$ ) We propose a simple scheme to detect the nontrivial topology of Weyl points based on momentumresolved Rabi spectroscopy and time-of-flight imaging. Surprisingly, the additional trivial band near the Weyl points can serve as a reference which greatly simplifies the detection pulse sequence.

\section{THE MODEL}

We consider a simple experimental setup shown in Fig. 1a, which contains a 1D superlattice along the $z$ direction with 3 sites in each unit cell and is free in $2 \mathrm{D}$ $x y$-plane (i.e., no transverse lattices). The detunings between different sites in the unit cell are large and the bare tunnelings are suppressed significantly. We introduce the Raman-assisted tunnelings using three Raman lasers [46, 47], with each site in the unit cell addressed by one and only one Raman laser whose frequency difference is chosen to match the lattice site detuning [see Fig. 1b]. The wave vectors should have nonzero components along $z$ to induce momentum kicks which is needed to generate the tunneling between neighbour sites. The Ramanassisted transitions acquire transverse momentum kicks that are determined by the transverse components of the wave vectors.

We adopt the tight-binding approach and expand the wave function as $|\Psi(\mathbf{r})\rangle=\sum_{j} a_{j}(x, y)\left|W_{j}(z)\right\rangle$, with $\left|W_{j}(z)\right\rangle$ the Wannier function for site $j$ in the $z$ direc- tion. The pseudo-spin operators in each unit cell are denoted as $b_{l, \downarrow}(x, y)=a_{3 l}(x, y), b_{l, 0}(x, y)=a_{3 l-1}(x, y)$, $b_{l, \uparrow}(x, y)=a_{3 l-2}(x, y)$ with $l$ the unit-cell index. The detunings between them are $\Delta_{\uparrow}, \Delta_{\downarrow}$, and $\Delta_{\uparrow} \pm \Delta_{\downarrow}$ (see Fig. 1), which are much larger than the bare nearest neighbor tunneling. Resonance tunnelings between neighbor lattice sites are induced by three Raman lasers with frequencies $\omega_{\uparrow}, \omega_{0}, \omega_{\downarrow}$, satisfying $\omega_{\uparrow}-\omega_{0} \simeq \Delta_{\uparrow}$, and $\omega_{\downarrow}-\omega_{0} \simeq \Delta_{\downarrow}$. The pseudo-spin state $|s\rangle$ is addressed by the laser with frequency $\omega_{s}(s=\uparrow, 0, \downarrow)$, which induce both intra- and inter-unit-cell tunnelings. The Ramanlaser wave vectors $\mathbf{K}_{s}=\mathbf{K}_{z, s}+\mathbf{K}_{\perp, s}$ (with $\mathbf{K}_{z, s} \neq \mathbf{K}_{z, s^{\prime}}$ for $s \neq s^{\prime}$ ) have nonzero components along both longitudinal and transverse directions, and the latter induces transverse spin-orbit couplings. The single-particle Hamiltonian in the rotating frame is

$\mathcal{H}=\sum_{l, s}\left(\frac{\mathbf{k}_{\perp}^{2}}{2 m}+\delta_{s}\right)|l, s\rangle\left\langle l, s\left|+\sum_{l, s ; l^{\prime}, s^{\prime}} J_{l, s ; l^{\prime}, s^{\prime}}\right| l, s\right\rangle\left\langle l^{\prime}, s^{\prime}\right|$,

where $|l, s\rangle$ is the single-particle state at unit-cell $l$ with spin $s$, the non-zero Raman-assisted tunnelings are $J_{l, 0 ; l, \uparrow}=J_{1} e^{i\left(\mathbf{K}_{\uparrow} \cdot \mathbf{r}_{l \uparrow}-\mathbf{K}_{0} \cdot \mathbf{r}_{l 0}\right)}, J_{l, \downarrow ; l, 0}=J_{2} e^{i\left(\mathbf{K}_{0} \cdot \mathbf{r}_{l 0}-\mathbf{K}_{\downarrow} \cdot \mathbf{r}_{l \downarrow}\right)}$ and $J_{l+1, \uparrow ; l, \downarrow}=J_{3} e^{i\left(\mathbf{K}_{\downarrow} \cdot \mathbf{r}_{\downarrow \downarrow}-\mathbf{K}_{\uparrow} \cdot \mathbf{r}_{\imath \uparrow}\right)}$ with $\mathbf{r}_{l s}=\left(x, y, z_{l s}\right)$ the coordinates of atoms at site $(l, s)$. $\delta_{s}$ is the corresponding detunings and $\mathbf{k}_{\perp}=\left(k_{x}, k_{y}\right)$ is the transverse momentum.

In the quasi-momentum frame after the transformation $|l, s\rangle \rightarrow e^{i \mathbf{K}_{s} \cdot \mathbf{r}_{l s}}|l, s\rangle$, we obtain the Hamiltonian

$$
\begin{aligned}
\mathcal{H}= & \sum_{l, s}\left[\frac{\left(\mathbf{k}_{\perp}-\mathbf{K}_{\perp, s}\right)^{2}}{2 m}+\delta_{s}\right]|l, s\rangle\langle l, s| \\
& +\sum_{l}\left(J_{1}|l, 0\rangle\left\langle l, \uparrow\left|+J_{2}\right| l, \downarrow\right\rangle\langle l, 0|\right. \\
& \left.+J_{3}|l+1, \uparrow\rangle\langle l, \downarrow|+\text { h.c. }\right),
\end{aligned}
$$

where $\mathbf{K}_{\perp, s}$ corresponds to the transverse momentum kick by the $s$-th Raman laser, which gives the $2 \mathrm{D}$ spinorbit coupling strengths in the transverse direction. The Hamiltonian in the Bloch momentum space is

$$
H_{\mathbf{k}}=\sum_{s}\left[\frac{\left(\mathbf{k}_{\perp}-\mathbf{K}_{\perp, s}\right)^{2}}{2 m}+\delta_{s}\right]|s\rangle\left\langle s\left|+\sum_{s \neq s^{\prime}} J_{s, s^{\prime}}(\mathbf{k})\right| s\right\rangle\left\langle s^{\prime}\right| .
$$

The intra-unit-cell couplings are $J_{\uparrow, 0}(\mathbf{k}) \equiv J_{0, \uparrow}^{*}(\mathbf{k})=J_{1}$ and $J_{0, \downarrow}(\mathbf{k}) \equiv J_{\downarrow, 0}^{*}(\mathbf{k})=J_{2}$, the inter-unit-cell coupling is $J_{\downarrow, \uparrow}(\mathbf{k})=J_{\uparrow, \downarrow}^{*}(\mathbf{k})=J_{3} e^{i k_{z}}$. In general, the tunnelling coefficients $J_{1,2,3}$ are complex whose phase are determined by the global phases of the Raman lasers. However, we notice that these phases are unimportant and can be gauged out by absorbing them in to the definition of the pseudospin state on each site, and there is no need for fine-tuning of the Raman-laser phases. We can simply set $J_{1,2,3}$ to be real (we will assume all $J_{1,2,3}$ positive unless 

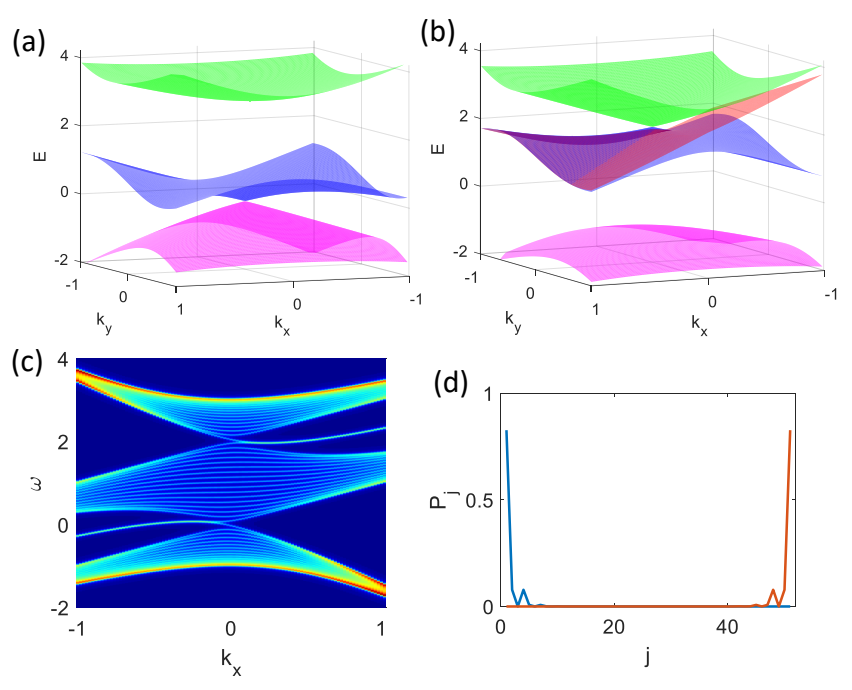

FIG. 2: (a) Band structure at $k_{z}=0$ with Weyl point $W_{1}$ between two lower bands. (b) Band structure at $k_{z}=\pi$ with Weyl point $W_{2}$ between two upper bands. The mid gap band shows the surface state dispersion on $z=0$ when open boundary conditions are considered. (c) Surface spectrum densities $\operatorname{Im} \frac{\gamma}{\pi} \frac{1}{\omega-H-i \gamma}$ at different frequencies and momenta, with $\gamma=0.02$ and $k_{y}=0$. The two surface arcs on the two surfaces coincide. (d) Typical distribution $\left(P_{j}\right)$ of the surface states along the open direction $z$, with $j$ the site index. $\frac{\mathbf{k}_{\perp}^{2}}{2 m}$ is dropped when plotting the band structures. Other parameters are $J_{1}=J_{2}=J_{3}=1, \mathbf{K}_{\perp, \uparrow}=(-1 / 2, \sqrt{3} / 2)$, $\mathbf{K}_{\perp, 0}=(1,0), \mathbf{K}_{\perp, \downarrow}=(-1 / 2,-\sqrt{3} / 2), \delta_{\uparrow}=\delta_{0}=\delta_{\downarrow}=0$.

otherwise state).

The Hamiltonian in Eq. (3) supports two robust Weyl points when all three couplings $J_{1,2,3}$ are nonzero and the three points $\mathbf{K}_{\perp, s}=\left(K_{x, s}, K_{y, s}\right)$ are non-collinear (see Appendix A). In this paper, we are interested in the Weyl physics where the three points $\mathbf{K}_{\perp, s}=\left(K_{x, s}, K_{y, s}\right)$ form a triangle (i.e., they are non-collinear). So, we can denote $\mathbf{Q}$ as the triangle's circumcenter and set $\mathbf{Q}$ as the origin of quasi-momentum frame by a transformation $|l, s\rangle \rightarrow e^{-i \mathbf{Q} \cdot \mathbf{r}_{l s}}|l, s\rangle$, the Hamiltonian reads

$H_{\mathbf{k}}=\sum_{s}\left[\frac{\left(\mathbf{k}_{\perp}-\mathbf{K}_{\perp, s}^{\prime}\right)^{2}}{2 m}+\delta_{s}\right]|s\rangle\left\langle s\left|+\sum_{s \neq s^{\prime}} J_{s, s^{\prime}}(\mathbf{k})\right| s\right\rangle\left\langle s^{\prime}\right|$

with $\mathbf{K}_{\perp, s}^{\prime}=\mathbf{K}_{\perp, s}-\mathbf{Q}$. Therefore, we have $\left|\mathbf{K}_{\perp, s}^{\prime}\right| \equiv$ $k_{R, \perp}$ for the three spin states $s$. We will set the momentum and energy unit as $k_{R, \perp}$ and $\frac{k_{R, \perp}^{2}}{2 m}=1$, and consider that the three points $\mathbf{K}_{\perp, s}^{\prime}=\left(K_{x, s}^{\prime}, K_{y, s}^{\prime}\right)$ changes on a unit circle (i.e., the three points $\mathbf{K}_{\perp, s}=\left(K_{x, s}, K_{y, s}\right)$ changes on a unit circle centered at $\mathbf{Q})$. In the following, we will focus on the Hamiltonian Eq. (4) and drop the prime symbol in $\mathbf{K}_{\perp, s}^{\prime}$ for simplicity.

We denote the two robust Weyl points as $W_{1}$ and $W_{2}$ at $\left[k_{x}^{W_{1}}, k_{y}^{W_{1}}, 0\right]$ and $\left[k_{x}^{W_{2}}, k_{y}^{W_{2}}, \pi\right]$. The Weyl point $W_{1}$ corresponds to the degeneracy between two lower bands
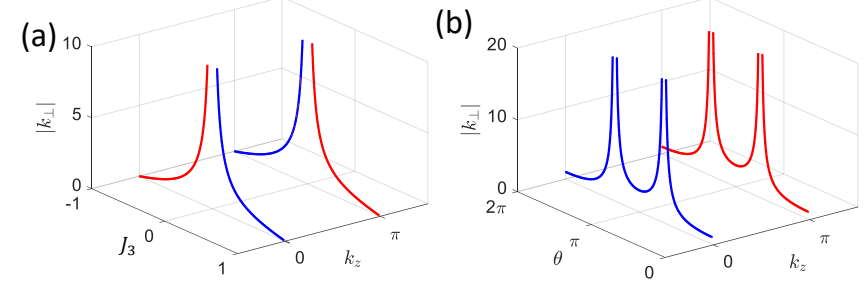

FIG. 3: (a) The trajectory of Weyl points $W_{1}$ (blue line) and $W_{2}$ (red line) as the inter-unit-cell tunneling varies across zero. $J_{1}=J_{2}=1, \mathbf{K}_{\perp, \uparrow}=(-1 / 2, \sqrt{3} / 2), \mathbf{K}_{\perp, 0}=(1,0)$, $\mathbf{K}_{\perp, \downarrow}=(-1 / 2,-\sqrt{3} / 2)$. Two Weyl points shift to infinite momenta at $J_{3}=0$. (b) The trajectories of Weyl points $W_{1}$ (blue line) and $W_{2}$ (red line) when $\mathbf{K}_{\perp, 0}=(\cos \theta, \sin \theta)$ rotates in the $k_{x}-k_{y}$ plane, $\mathbf{K}_{\perp, \uparrow}=(-1 / 2, \sqrt{3} / 2), \mathbf{K}_{\perp, \downarrow}=$ $(-1 / 2,-\sqrt{3} / 2), J_{1}=J_{2}=2 J_{3}=1$. The two Weyl points shift to infinite momenta at $\theta=2 \pi / 3$ and $4 \pi / 3$ where $\mathbf{K}_{\perp, 0}$ coincides with $\mathbf{K}_{\perp, \uparrow}$ and $\mathbf{K}_{\perp, \downarrow}$ respectively.

while $W_{2}$ for two upper bands. $W_{1}$ and $W_{2}$ are related to different bands, therefore they cannot annihilate with each other. Any change in system parameters only shifts the positions of the Weyl points. The typical band structures are shown in Figs. 2a and 2b, where two Weyl points are clearly shown. $\frac{\mathbf{k}_{\perp}^{2}}{2 m}$ is dropped when plotting the band structures in Fig. 2, our system is not a semimetal and the dispersion relation at higher values of $\mathbf{k}_{\perp}$ goes up in energy.

\section{SURFACE ARCS AND WEYL POINT TRAJECTORIES}

In general, Weyl points between any two bands should appear in pairs for a 3D lattice system because the Brillouin zone is a closed manifold without boundary [23-26]. Our system is free in the $x y$ plane, therefore we could have only one Weyl point between two bands since the momentum space is an open manifold that may have nonvanishing flux on the boundary (at infinite $k_{x}$ and $k_{y}$ ). This can be seen by looking at the surface arcs which can only start (end) at the Weyl points. In Figs. 2b and $2 \mathrm{c}$, we plot the surface arcs with an open boundary condition along the $z$-direction. Each boundary (left and right) gives a surface arc which connects the Weyl point to infinite momenta. Shown in Fig. 2d are the distributions of the surface states that are well localized at the boundary.

There is only one Weyl point between two neighbor bands, which can annihilate with its virtual partner only when it is shifted to infinity at certain critical system parameters. The only symmetry required here is the lattice symmetry along the $z$-direction, thus the Weyl points are very robust against system disorders. Shown in Fig. 3 are the trajectories of two Weyl points $W_{1}$ and $W_{2}$ as functions of $J_{1,2,3}$ and $\mathbf{K}_{\perp, s}$. As one of the couplings $J_{1,2,3}$ 
changes across the critical value 0 (from positive to negative), two Weyl points first disappear then reappear at infinity $\left(\mathbf{k}_{\perp} \rightarrow \infty\right)$, with $k_{z}$ changing from $0(\pi)$ to $\pi(0)$ for Weyl point $W_{1}\left(W_{2}\right)$. The two Weyl points move similarly as $\mathbf{K}_{\perp, s}-\mathbf{K}_{\perp, s^{\prime}}$ changes across the critical value 0 , except that $k_{z}$ is fixed for both of them. The Ramanlaser phases are irrelevant since the phases of $J_{1,2,3}$ do not affect the band structure (e.g., the phase of $J_{3}$ only induces a global shift of all bands along $k_{z}$ ). Finally, the pseudospin is represented by different superlattice sites on the same atomic hyperfine state, making the tunnelings $J_{1,2,3}$ insensitive to laser polarizations.

\section{BERRY FLUX AND SPIN TEXTURES}

The topological properties of the Weyl point can be characterized by the first Chern number $[3,4]$

$$
\mathcal{C}_{n}=\frac{1}{2 \pi} \oint_{\mathbf{S}} \nabla_{\mathbf{k}} \times \mathcal{A}_{n}(\mathbf{k}) \cdot d \mathbf{S},
$$

where $\mathbf{S}$ is a momentum-space surface enclosing the Weyl point, and $\mathcal{A}_{n}(\mathbf{k})=i\left\langle u_{n}(\mathbf{k})\left|\nabla_{\mathbf{k}}\right| u_{n}(\mathbf{k})\right\rangle$ is the Berry connection, with $\left|u_{n}(\mathbf{k})\right\rangle$ the eigenvector (Bloch wave function) of the $n$-th band. $\mathcal{C}_{n}= \pm 1$ indicates that the Berry curvature (flux) $\boldsymbol{\Omega}_{n}(\mathbf{k})=\nabla_{\mathbf{k}} \times \mathcal{A}_{n}(\mathbf{k})$ on the closed surface $\mathbf{S}$ is quantized, revealing the synthetic magnetic monopole behavior. The distribution of Berry curvatures around $W_{1}$ is shown in Fig. 4a (Berry curvatures for different bands and different surface $\mathbf{S}$ can be found in Appendix B), yielding $\mathbf{C} \equiv\left[\mathcal{C}_{1}, \mathcal{C}_{2}, \mathcal{C}_{3}\right]=[-1,1,0]$ for the Weyl point $W_{1}$, and $\mathbf{C}=[0,1,-1]$ for $W_{2}$. When both $W_{1}$ and $W_{2}$ are enclosed by $\mathbf{S}$, we have $\mathbf{C}=[-1,2,-1]$. Notice that the Chern numbers remain unchanged even when the radius of the $\mathbf{S}$ approaches infinity, which explains why the surface arcs are connected to infinity momenta, indicating that there is another pair of Weyl points with opposite charges.

For a spin- $1 / 2$ system, the quantum state is uniquely represented by a point on the Bloch sphere whose coordinates are given by the expectation value of spin vector $\langle\mathbf{F}\rangle$. As momentum $\mathbf{k}$ runs over a surface enclosing a Weyl point in such spin- $1 / 2$ system, $\langle\mathbf{F}\rangle$ also covers the Bloch sphere once, and the Berry flux is given by the solid angle on the Bloch sphere. Spin-1 (and higher) quantum states are quite different: first, its quantum state is not uniquely represented by the spin vector $\langle\mathbf{F}\rangle$; and second, $\langle\mathbf{F}\rangle$ is not confined to the surface of the Bloch sphere, and could be anywhere on or inside the Bloch sphere. For high spins $(\geq 1)$, the spin moments contain both spin vectors and spin tensors. The spin- 1 quantum state can be uniquely represented by the combination of the spin vector $\langle\mathbf{F}\rangle$ and a rank-2 spin tensor $T$ with elements $T_{i j}=\left\langle\frac{F_{i} F_{j}+F_{j} F_{i}}{2}\right\rangle-\left\langle F_{i}\right\rangle\left\langle F_{j}\right\rangle$, which is geometrically characterized by an ellipsoid. The ellipsoid is fully determined
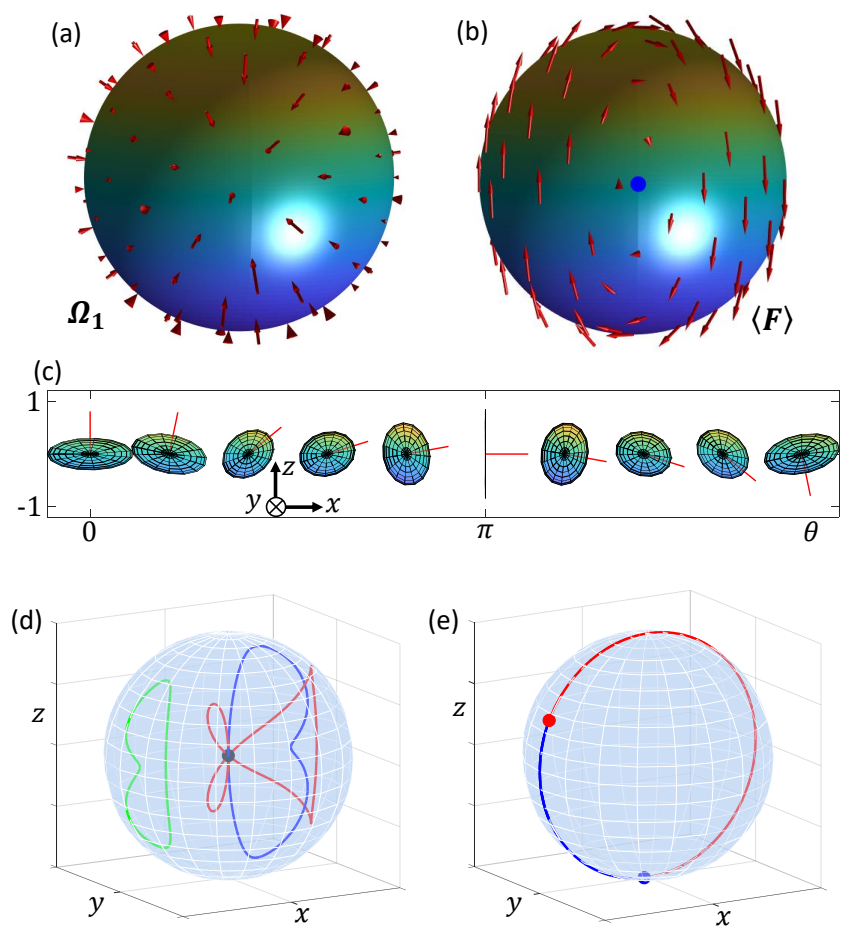

FIG. 4: (a) and (b) Berry flux and spin vector distributions on the sphere $\mathbf{S}$ enclosing Weyl point $W_{1}$. (c) Spin tensor distribution as $\mathbf{k}$ varies on the loop $\mathcal{L}$. The red lines show the direction of the ellipsoid's one axis, which is rotated by $\pi$ along the loop. (d) The spin vector trajectories for the loop $\mathcal{L}$ for all three bands. $\langle\mathbf{F}\rangle$ would cross the center of the Bloch sphere only for the two lower non-trivial bands (pink and blue lines). (e) The trajectories of the two Majorana stars on the Bloch sphere for the loop $\mathcal{L}$ (the blue and red dots show their initial positions). They exchange their positions and together give rise to a $\pi$ Berry phase. The radius of sphere $\mathbf{S}$ is $r_{S}=1$ and other parameters are the same as in Fig. 2.

by its three axes, whose lengths and directions are given by the eigenvectors and the square root of the eigenvalues of $T$, respectively [52-54]. The topology of the Weyl point in our spin-1 system should be characterized by the geometries of both the spin vector and tensor textures, which are fundamentally different from spin- $1 / 2$ systems.

An arbitrary spin-1 quantum state $|\Psi\rangle$ can be characterized by four parameters $F, \phi_{F}, \theta_{F}, \phi_{T}$, where $F=|\langle\mathbf{F}\rangle|$ is the spin-vector length, $\phi_{F}, \theta_{F}$ determine the direction of the spin vector and $\phi_{T}$ gives the relative rotation of the spin-tensor-ellipsoid with respect to the spin vector [5254]. This is because, for a given $\langle\mathbf{F}\rangle$ (i.e., $F, \phi_{F}, \theta_{F}$ ), the size of the spin-tensor-ellipsoid is also fixed with three axis lengths $\sqrt{1-F^{2}}, \sqrt{\frac{1 \pm \sqrt{1-F^{2}}}{2}}$. Moreover, the axis with length $\sqrt{1-F^{2}}$ has the same direction with $\langle\mathbf{F}\rangle$, and $\phi_{T}$ gives the direction of the other two axes with length $\sqrt{\frac{1 \pm \sqrt{1-F^{2}}}{2}}$, which fixes the orientation of the el- 
lipsoid [52-54]. In particular, we have

$$
\left|\Psi\left(F, \phi_{F}, \theta_{F}, \phi_{T}\right)\right\rangle=D\left(\phi_{F}, \theta_{F}, \phi_{T}\right)\left[\begin{array}{c}
\sqrt{\frac{1+F}{2}} \\
0 \\
\sqrt{\frac{1-F}{2}}
\end{array}\right]
$$

with $D\left(\phi_{F}, \theta_{F}, \phi_{T}\right)=e^{-i F_{z} \phi_{F}} e^{-i F_{y} \theta_{F}} e^{-i F_{z} \phi_{T}}$. Let's consider an infinitesimal sphere $\mathbf{S}$ enclosing the Weyl point, the state of the third far gapped band remains unchanged on the whole sphere since the sphere is infinitesimal. We can denote the third-band state at the Weyl point as $\left|u_{3}^{0}\right\rangle=\left|\Psi\left(F, \phi_{F}, \theta_{F}, \phi_{T}\right)\right\rangle$. We first consider $F \neq 0$, there exits one and only one state $\left|u_{2}^{0}\right\rangle=\left|\Psi\left(F, \phi_{F}, \theta_{F}+\pi, \phi_{T}+\pi\right)\right\rangle$ satisfying $\left\langle u_{3}^{0} \mid u_{2}^{0}\right\rangle=0$ and $\left\langle u_{3}^{0}|\mathbf{F}| u_{3}^{0}\right\rangle=-\left\langle u_{2}^{0}|\mathbf{F}| u_{2}^{0}\right\rangle$. Notice that $\mathbf{F}$ is traceless $\operatorname{tr}(\mathbf{F})=0$, therefore, there exist one and only one state satisfying $\left\langle u_{3}^{0} \mid u_{1}^{0}\right\rangle=0$ and $\left\langle u_{1}^{0}|\mathbf{F}| u_{1}^{0}\right\rangle=0$. Naturally, we also have $\left\langle u_{2}^{0} \mid u_{1}^{0}\right\rangle=0$. The Weyl point is characterized by an effective spin- $1 / 2$ system spanned by states $\left|u_{1}^{0}\right\rangle$ and $\left|u_{2}^{0}\right\rangle$. As the momentum changes over the sphere $\mathbf{S}$, the eigenstate of the two Weyl bands changes on the Bloch sphere spanned by $\left|u_{1}^{0}\right\rangle$ and $\left|u_{2}^{0}\right\rangle$. The Chern number counts the times the eigenstate covers the Bloch sphere. $\left|u_{1}^{0}\right\rangle$ is the only state on the Bloch sphere that gives vanishing $\langle\mathbf{F}\rangle$, therefore, the Chern number is odd (even) if and only if there are odd (even) numbers vanishing points of the spin vector. For the special case with $F=0$, it can be shown that $\langle\mathbf{F}\rangle$ can vanish on a loop (a great circle coinciding with the prime meridian) instead of a point on the Bloch sphere, and thus the Chern number is odd (even) if and only if there are odd (even) numbers of vanishing loops of the spin vector. The spin vectors form a vortex (change their sign) around the vanishing point (across the vanishing loop). Therefore, the spin-vector vanishing points and loops are topological structures that can only change abruptly (e.g., at a gap closing), and they will remain unchanged under smooth deformations (e.g., enlarging the sphere $\mathbf{S}$ ).

In Fig. 4b, we show the spin vector distribution $\langle\mathbf{F}\rangle$ (calculated for the lowest band) around $W_{1}$ (spin textures for different bands and different surfaces $\mathbf{S}$ can be found in Appendix B). We see that the spin vector may vanish at one certain point (blue dot), around which spin vortex emerges. Though the Chern number of the Weyl point can be obtained by the number of spin vortices, the Berry phase along a loop (i.e., Berry flux on a surface enclosed by the loop) in the momentum space (or other parameter space) is given by the generalized solid angles involving contributions from both spin vectors and tensors on or inside the Bloch sphere (see Appendix C).

To illustrate how the spin tensor is distributed around $W_{1}$, we consider a loop on $\mathbf{S}$, and study how the ellipsoid rotates along it. Fig. 4c shows the spin tensor (with view direction along $y$ axis) for the first band along the loop $\mathcal{L}: k_{x}=r_{S} \cos \theta, k_{y}=r_{S} \sin \theta, k_{z}=0$ with $r_{S}$ the radius and $\theta$ varies from 0 to $2 \pi$ (i.e., the equator of $\mathbf{S}$ ). As $\theta$ increases from 0 to $2 \pi$, the ellipsoid is reduced to a $2 \mathrm{D}$ disk at $\theta=\pi$, where the spin vector crosses the center of the Bloch sphere as shown in Fig. $4 \mathrm{~d}(\langle\mathbf{F}\rangle$ vanishes and changes the sign). Along the loop $\mathcal{L}$, the spin vector is confined in the $F_{x}-F_{z}$ plane, which gives rise to zero solid angle. Beside the size oscillation, the orientation of the spin tensor ellipsoid rotates around the $y$-axis by $\pi$, which correspond to a $\pi$ Berry phase along $\mathcal{L}$ due to the fact that the Weyl point reduces to a Dirac point in the $k_{z}=0$ plane. Along the loop $\mathcal{L}$, one of the ellipsoid's axes is fixed along $y$ direction, and its length is around 0.85 which changes slightly with $\theta$. Similar spin tensor rotation can be obtained for the second band. However, the spin vector crosses the center of the Bloch sphere three times on the loop $\mathcal{L}$, leading to three spin-vector vortices on $\mathbf{S}$.

The nontrivial topology of the Weyl points can also be captured by the trajectories of two Majorana stars (an unordered pair of points on the Bloch sphere) [55]. The Berry flux is given by the correlated solid angle of the two Majorana stars (see Appendix D). For the loop $\mathcal{L}$ considered in Fig. 4c, we find that the Majorana stars are confined in the $y=0$ plane on the Bloch sphere. As $\theta$ increases, similar to the spin-tensor ellipsoid, the Majorana stars also rotate with respect to $y$-axis. Instead of going back to their originate positions after one circle, two Majorana stars exchange as shown in Fig. 4e, leading to a solid angle $\pi$.

\section{IMPLEMENTATION AND DETECTION}

Our scheme does not rely on atomic hyperfine level structure, and is applicable to both alkaline atoms (e.g., Lithium, potassium) and alkaline-earth(-like) atoms (e.g., strontium, ytterbium) [56-59]. The triple-well superlattice could be realized by a superposition of two lattice potentials with one of them having a tripled period,

$$
V(z)=V_{1} \cos ^{2}\left(k_{L} z\right)+V_{2} \cos ^{2}\left(k_{L} z / 3+\phi_{L}\right) .
$$

Using optical frequency tripling [60, 61], such two lattice potential can be obtained with tunable relative phase $\phi_{L}$, similar as the double well superlattice based on the optical frequency doubling in recent experiments [62-65]. Alternatively, it can also be realized using lasers with the same wavelength, while the long-period lattice is formed by two beams intersecting with an angle $\theta=2 \arcsin \frac{1}{3}$. By choosing proper lattice strengths $V_{1}, V_{2}$ and the relative phase $\phi_{L}$, the detunings between different sites in a unit cell is tuned to be much larger than the bare nearest neighbor tunneling. The tunnelings can be restored using resonant Raman couplings, as demonstrated by recent experiments in the study of gauge field and supersolidity. 
The linear dispersion of the Weyl point can be detected using momentum-resolved radio-frequency (rf) spectroscopy [66], which has been widely used to study low-energy excitation spectrum and quasiparticles in superfluids and superconductors. Based on energy and momentum conservation, the Weyl point dispersion can be extracted from the time-of-flight absorption image after the rf pulse. In general, direct measurement of non-trivial Berry curvatures and spin textures of Weyl points is very challenging, and simple schemes for probing Weyl-point topology are still elusive. Here we propose that the detection can be realized by the momentum-resolved Rabi spectroscopy [67] with simple pulse sequences. Surprisingly, the simplification comes from the presence of the third band near the Weyl point for our spin-1 system. First, the system is initialized into the pseudo-spin state $|s\rangle$, then the Raman lasers are turned on. By simply measuring the evolution of atom population on state $\left|s^{\prime}\right\rangle$ at each $\mathbf{k}$, the Bloch wave-function (and thereby the Berry curvatures and spin textures) near the Weyl points can be extracted. There is no need to measure the population on different basis as required for spin- $1 / 2$ systems. This is because, beside two non-trivial bands, there is a far detuned trivial band near the Weyl point, which can serve as a reference band, allowing us to determine both amplitudes and phases of the Bloch functions for two non-trivial bands. In realistic experiments, the population of each spin state $|s\rangle$ at each $\mathbf{k}$ can be measured using a pseudospin Stern-Gerlach effect followed by the time-of-flight imaging [64, 65].

It has been demonstrated that, for a spin- $1 / 2$ system, the Bloch wave function, which directly determines the Berry curvatures and spin textures, can be extracted from the momentum-resolved Rabi spectroscopy realized by proper choice of laser pulse sequences [51]. Surprisingly, for our spin-1 system, the presence of a third band would greatly simplify the pulse sequence. The Bloch wavefunction of the $n$-th band with energy $E_{n}(\mathbf{k})$ is

$$
\left|u_{n}(\mathbf{k})\right\rangle=\sum_{s} U_{n, s}(\mathbf{k})|\mathbf{k}, s\rangle
$$

with $U_{n, s}(\mathbf{k})$ the element of the unitary matrix $U$. Consider an initial state $|\Psi(0)\rangle=|\mathbf{k}, s\rangle$, the Hamiltonian would induce a Rabi oscillation and give a final state at time $\tau$

$$
|\Psi(\tau)\rangle=\sum_{n} e^{-i E_{n}(\mathbf{k}) \tau} U_{n, s}^{*}(\mathbf{k})\left|u_{n}(\mathbf{k})\right\rangle .
$$

In the following, we prove that the Bloch wave function can be obtained by simply measuring the final state in the spin basis $\{|\mathbf{k}, s\rangle\}$ with $s=\uparrow, 0, \downarrow$. Thanks to the presence of the third band, the detecting scheme is simpler comparing with the spin-1/2 system (where measurements in various bases and thus additional precisely controlled pulses are required) [51].
The population on state $\left|\mathbf{k}, s^{\prime}\right\rangle$ of the final state is

$$
P_{s, s^{\prime}}(\mathbf{k}, \tau)=\left|\sum_{n} e^{-i E_{n}(\mathbf{k}) \tau} U_{n, s}^{*}(\mathbf{k}) U_{n, s^{\prime}}(\mathbf{k})\right|^{2} .
$$

We define the averaged population $\bar{P}_{s, s^{\prime}}$ as

$$
\bar{P}_{s, s^{\prime}}=\frac{P_{s, s^{\prime}}+P_{s^{\prime}, s}}{2} \text {. }
$$

Use the Fourier analysis in the time domain $\bar{P}_{s, s^{\prime}}(\mathbf{k}, \omega)=$ $\int d \tau \bar{P}_{s, s^{\prime}}(\mathbf{k}, \tau) \cos (\omega \tau)$, we obtain

$$
\begin{aligned}
& \bar{P}_{s, s^{\prime}}(\mathbf{k}, \omega)=\sum_{n<n^{\prime}}\left[\left|U_{n, s}^{*} U_{n, s^{\prime}} U_{n^{\prime}, s^{\prime}}^{*} U_{n^{\prime}, s}\right|\right. \\
& \left.\times \cos \left(\phi_{n ; s, s^{\prime}}+\phi_{n^{\prime} ; s^{\prime}, s}\right) \delta\left(E_{n^{\prime}}-E_{n}-\omega\right)\right],
\end{aligned}
$$

where relative phase $\phi_{n ; s, s^{\prime}}=\phi_{n ; s^{\prime}}-\phi_{n ; s}$ with $\phi_{n ; s}=$ $\arg \left[U_{n, s}\right]$. For $s^{\prime}=s$, we can easily obtain the amplitude of the matrix elements $\left|U_{n, s}\right|$ based on $\bar{P}_{s, s^{\prime}}(\mathbf{k}, \omega)$ and the unitary property of matrix $U$. Extracting the phase information is, however, a little bit tricky. For Weyl points in a spin- $1 / 2$ system, it is impossible to determine the phase $\phi_{n ; s, s^{\prime}}$ from $\bar{P}_{s, s^{\prime}}(\mathbf{k}, \omega)$ since both $\phi_{n ; s, s^{\prime}}$ and $\phi_{n^{\prime} ; s^{\prime}, s}$ changes rapidly near the Weyl point and one can only obtain their summation $\phi_{n ; s, s^{\prime}}+\phi_{n^{\prime} ; s^{\prime}, s}$ (not to mention that this summation usually vanishes). However, for a spin-1 system, the third band can serve as a reference which allows the determination of the phases for the other two bands.

To show how our detecting scheme works, we focus our discussion on Weyl point $W_{1}$ in the following. In the vicinity of Weyl point $W_{1}$, the Bloch wavefunctions possess non-trivial topology due to the degeneracy for two lower bands, but are trivial and almost unchanged for the highest band. Near the frequency $\omega=E_{3}-E_{n}$, we have

$$
\bar{P}_{s, s^{\prime}} \propto\left|U_{n, s}^{*} U_{n, s^{\prime}} U_{3, s^{\prime}}^{*} U_{3, s}\right| \cos \left(\phi_{n ; s, s^{\prime}}-\phi_{3 ; s, s^{\prime}}\right),
$$

where $\phi_{3 ; s, s^{\prime}}$ is a constant near the Weyl point, and can be set to zero by absorbing it to the definition of $|\mathbf{k}, s\rangle$. Therefore we obtain the relative phase $\phi_{n ; s, s^{\prime}}$ (with $n=1,2$ ) for the two non-trivial bands through measuring $\bar{P}_{s, s^{\prime}}$. In fact, even the phase $\phi_{3 ; s, s^{\prime}}$ is not a constant, the topologies of the Bloch functions are not affected by absorbing $\phi_{3 ; s, s^{\prime}}$ into the definition of $|\mathbf{k}, s\rangle$, as long as $\phi_{3 ; s, s^{\prime}}$ is a non-singular and smooth function near the Weyl point $W_{1}$. $\left|U_{n, s}\right|$ and $\phi_{n ; s, s^{\prime}}$ can be uniquely determined in a way such that the Bloch wave function is smooth.

The measured relative phase, which is used to extract the Bloch wave function, is $\phi_{n ; s, s^{\prime}}^{M}=\phi_{n ; s, s^{\prime}}-\phi_{3 ; s, s^{\prime}}$. As a result, the measured Bloch wave function $\left|u_{n}^{M}(\mathbf{k})\right\rangle$ and the true Bloch wave function $\left|u_{n}(\mathbf{k})\right\rangle$ are related by a 
(a)

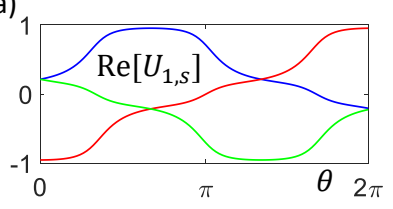

(b)

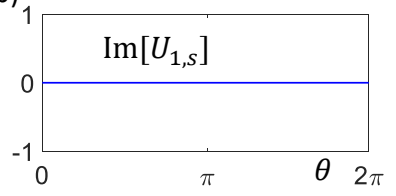

(c)

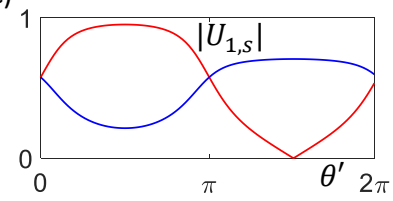

(d)

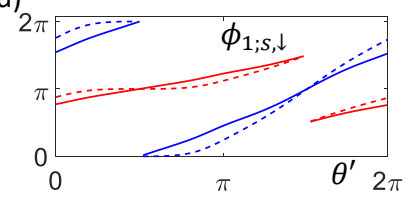

FIG. 5: (a) and (b) The real and imaginary parts of $U_{1, s}$ on loop $\mathcal{L}: k_{x}=\cos \theta, k_{y}=\sin \theta, k_{z}=0$ around $W_{1}$, with blue, red and green lines corresponding to the spin states $s=\uparrow$, $s=0$ and $s=\downarrow$, respectively. (c) and (d) The amplitude and phase of $U_{1, s}$ on loop $\mathcal{L}^{\prime}: k_{z}=\cos \theta, k_{x}=\sin \theta, k_{y}=0$, with blue and red lines corresponding to the spin states $s=\uparrow$ and $s=0$, respectively. The amplitude for $s=\downarrow$ is the same as that for $s=\uparrow$, and the phase is measured with respect to spin state $s=\downarrow$. In (d), dashed and solid lines correspond to the measured and true values, respectively. Other parameters are the same as in Fig. 4.

unitary transformation $\left|u_{n}^{M}(\mathbf{k})\right\rangle=e^{-i \hat{\Phi}}\left|u_{n}(\mathbf{k})\right\rangle$, with $\hat{\Phi}=$ $\operatorname{diag}\left\{\phi_{3 ; \uparrow}, \phi_{3 ; 0}, \phi_{3 ; \downarrow}\right\}$. The measured Chern number using $\left|u_{n}^{M}(\mathbf{k})\right\rangle$ is

$$
\mathcal{C}_{n}^{M}=\frac{1}{2 \pi} \oint_{\mathbf{S}}\left[\boldsymbol{\Omega}_{n}+\nabla_{\mathbf{k}} \times\left\langle u_{n}(\mathbf{k})|\hat{\chi}| u_{n}(\mathbf{k})\right\rangle\right] \cdot d \mathbf{S},
$$

with $\hat{\chi}=\nabla_{\mathbf{k}} \hat{\Phi}$. In the very vicinity of the Weyl point $W_{1}, \hat{\Phi}$ is a constant diagonal matrix, and the second term in the square brackets of the above equation vanishes. Therefore the measured Berry curvature and Chern number are the same as their true values. Far away from the Weyl point, $\hat{\Phi}$ becomes $\mathbf{k}$ dependent, and the measured Berry curvature may have small derivations from the true value, however, the measured Chern number is unaffected as long as $e^{-i \hat{\Phi}}$ is non-singular and smooth, which holds for our case when $\mathbf{S}$ only encloses one Weyl point $W_{1}$.

In Fig. 5, we show numerical results for the phases extracted from $\bar{P}_{s, s^{\prime}}$ and their true values obtained directly from the Hamiltonian on the two loops $\mathcal{L}$ and $\mathcal{L}^{\prime}$. For the loop $\mathcal{L}$, we always have $\phi_{n ; s, s^{\prime}}=0, \pi$, so the Bloch wavefunction can be extracted solely from $\left|U_{n, s}\right|$, while the phase can be determined simply by the continuous properties. For the loop $\mathcal{L}^{\prime}$ with a large radius $r_{S}=1$, we see small derivations of the measured relative phases from their true values.

In realistic experiments, the initialization is realized by first tuning the lattice potential such that the $s$-sites have the lowest energy in each unit cell, loading atoms to the pseudo-spin state $|s\rangle$, and then adiabatically tuning the potential to the desired superlattices. Next, we can turn on the Raman lasers and let the system evolve with

an interval $\tau$. The population of the final state on $s^{\prime}$ sites at each $\mathbf{k}$ (i.e., $\left.P_{s, s^{\prime}}(\mathbf{k}, \tau)\right)$ can be measured using a pseudospin Stern-Gerlach effect followed by the time-offlight imaging $[64,65]$.

\section{CONCLUSION}

In summary, we propose a simple scheme to realize robust Weyl points and probe their topology, using a 1D triple-well superlattice with transverse 2D SOC generated by three Raman lasers. The robustness against system parameters such as laser intensities, phases, polarizations and incident angles makes our scheme very flexible, and any fine-tuning or phase-locking techniques are not required. Moreover, we find that the spin- 1 Weyl point shows very interesting and topologically non-trivial spin (vector and tensor) textures that have fundamental differences from spin- $1 / 2$ systems. Thanks to the threeband structure, these non-trivial topologies can be detected using very simple pulse sequences. A straightforward generalization of our scheme is to consider higherorder degeneracies (e.g., three- or four-fold) [68-71] using even higher spins, which may be realized by using a superlattice with more sites in each unit-cell or by including atomic hyperfine states. Our scheme provides a simple yet powerful platform for exploring Weyl physics and related high-dimensional topological phenomena with ultracold atoms.

Acknowledgements: This work is supported by AFOSR (FA9550-16-1-0387), NSF (PHY-1806227), and ARO (W911NF-17-1-0128).

\section{Appendix}

\section{A. The Weyl point solution}

In the basis $\{|\uparrow\rangle,|0\rangle,|\downarrow\rangle\}$, the momentum space Hamiltonian is

$$
\begin{aligned}
H_{\mathbf{k}}= & {\left[\begin{array}{ccc}
\bar{\delta}_{\uparrow}-\frac{\mathbf{k}_{\perp} \cdot \mathbf{K}_{\perp, \uparrow}}{m} & J_{1} & J_{3} e^{i k_{z}} \\
J_{1}{ }^{i k_{z}} & \bar{\delta}_{0}-\frac{\mathbf{k}_{\perp} \cdot \mathbf{K}_{\perp, 0}}{m} & J_{2} \\
J_{3} e^{-i k_{z}} & J_{2} & \bar{\delta}_{\downarrow}-\frac{\mathbf{k}_{\perp} \cdot \mathbf{K}_{\perp, \downarrow}}{m}
\end{array}\right] } \\
& +\frac{\mathbf{k}_{\perp}^{2}}{2 m}
\end{aligned}
$$

with $\bar{\delta}_{s}=\delta_{s}+\frac{\mathbf{K}_{\perp, s}^{2}}{2 m}$. We can redefine the Fermi energy $E_{F}$ as the zero energy point, and rewrite the Hamiltonian as

$$
H_{\mathbf{k}}=\left[\begin{array}{ccc}
\bar{\delta}_{\uparrow}-\frac{\mathbf{k}_{\perp} \cdot \mathbf{K}_{\perp, \uparrow}}{m} & J_{1} & J_{3} e^{i k_{z}} \\
J_{1} & \bar{\delta}_{0}-\frac{\mathbf{k}_{\perp} \cdot \mathbf{K}_{\perp, 0}}{m} & J_{2} \\
J_{3} e^{-i k_{z}} & J_{2} & \bar{\delta}_{\downarrow}-\frac{\mathbf{k}_{\perp} \cdot \mathbf{K}_{\perp, \downarrow}}{m}
\end{array}\right]
$$



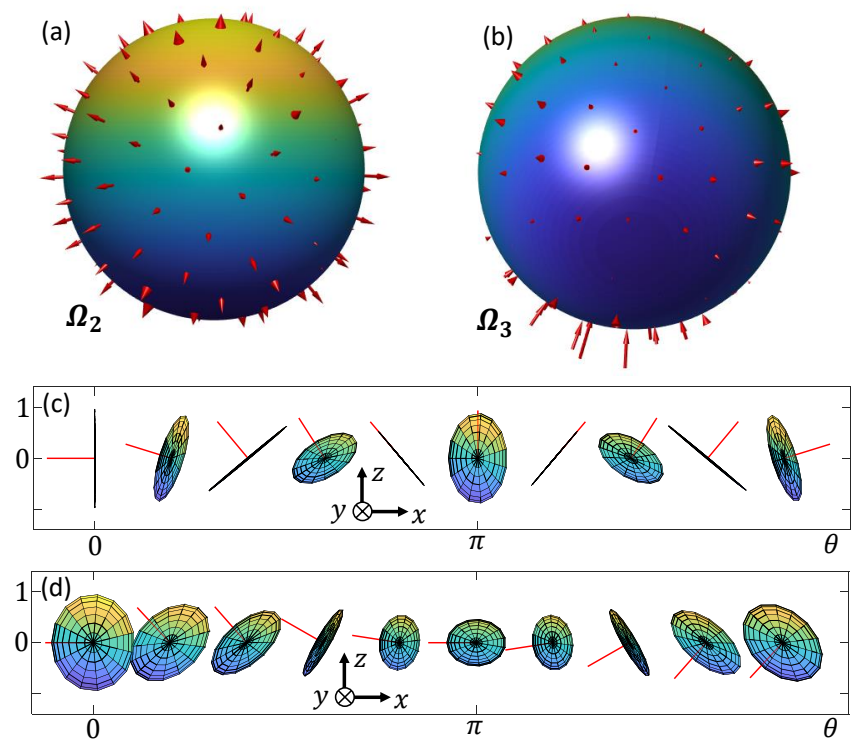

FIG. 6: (a) and (b) The Berry flux distributions on the surface enclosing Weyl point $W_{1}$ for the second and third bands, respectively. (c) and (d) Spin tensor distributions as $\mathbf{k}$ varies on the loop $\mathcal{L}$ for the second and third band, respectively. The red lines show the orientation of the ellipsoids, which are rotated by $\pi$ (0) for the second (third) band. Other parameters are the same as in Fig. 4.

$$
+\frac{\mathbf{k}_{\perp}^{2}}{2 m}-E_{F}
$$

The Weyl point corresponds to a two-fold degeneracy with zero energy, which requires that there exist a Fermi energy $E_{F}$ and a momentum $\mathbf{k}^{W}$ such that the above Hamiltonian is a rank-1 matrix. At the Weyl points, $E_{F}$ and $\mathbf{k}^{W}$ correspond to the solutions of the following equations

$$
\left\{\begin{array}{l}
\frac{\bar{\delta}_{\uparrow}-\frac{\mathbf{k}_{\perp} \cdot \mathbf{K}_{\perp, \uparrow}}{m}+E_{\perp}-E_{F}}{J_{1}}=\frac{J_{1}}{-\frac{\mathbf{k}_{\perp} \cdot \mathbf{K}_{\perp, 0}}{m}+E_{\perp}-E_{F}}=\frac{J_{3} e^{i k_{z}}}{J_{2}} \\
\frac{\bar{\delta}_{\uparrow}-\frac{\mathbf{k}_{\perp} \cdot \mathbf{K}_{\perp, \uparrow}}{m}+E_{\perp}-E_{F}}{J_{3} e^{-i k_{z}}}=\frac{J_{1}}{J_{2}}=\frac{J_{3} e^{i k_{z}}}{\bar{\delta}_{\downarrow}-\frac{\mathbf{k}_{\perp} \cdot \mathbf{K}_{\perp, \downarrow}}{m}+E_{\perp}-E_{F}} .
\end{array}\right.
$$

We have set $\bar{\delta}_{0}=0$ without loss of generality. Therefore, we have $k_{z}^{W}=0$ or $\pi$, and $\mathbf{k}_{\perp}^{W}$ is the solution of the equations

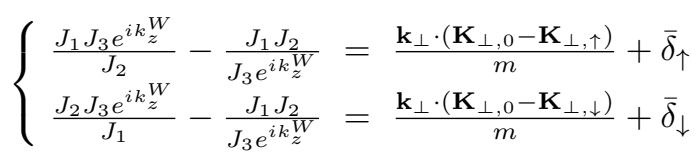

We always have solutions as long as $J_{1,2,3}$ are nonzero and $\mathbf{K}_{\perp, 0}-\mathbf{K}_{\perp, \uparrow}$ is not parallel with $\mathbf{K}_{\perp, 0}-\mathbf{K}_{\perp, \downarrow}$ (i.e., the three points $\mathbf{K}_{\perp, s}=\left(K_{x, s}, K_{y, s}\right)$ are not collinear), which share the same spirit as the recent study of Dirac degeneracy with 2D spin-orbit coupling [41, 42]. The fermi energy is given by $E_{F}=\frac{\left(\mathbf{k}_{\perp}^{W}\right)^{2}}{2 m}-\frac{J_{1} J_{2}}{J_{3} e^{i k W}}-\frac{\mathbf{k}_{\perp}^{W} \cdot \mathbf{K}_{\perp, 0}}{m}$. We would like to point out that, for pseudo-spin states repre-
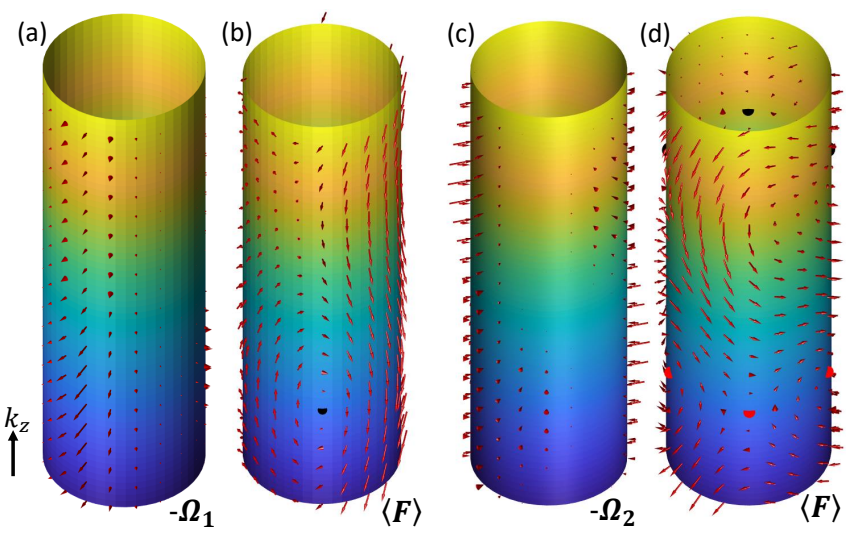

FIG. 7: (a) and (b) The Berry flux and spin vector distributions of the first band on the surface enclosing both Weyl points $W_{1}$ and $W_{2}$. A spin vortex (black dot) is located at $k_{z}=0$. (c) and (d) The Berry flux and Spin vector distributions of the second band on the surface enclosing both Weyl points $W_{1}$ and $W_{2}$. Six spin vortices (red and black dots) are located at $k_{z}=0$ and $k_{z}=\pi$. The radius of the cylinder is 1 , other parameters are the same as in Fig. 4.

sented by the atomic hyperfine levels as in [41, 42] (where the 2D Dirac degeneracy is sensitive to the Raman-laser polarizations), it is not easy to generalize the 2D Dirac degeneracy to 3D Weyl degeneracy.

\section{B. Berry flux and spin textures on different surfaces}

As we discussed in the main text, we have $\mathbf{C} \equiv$ $\left[\mathcal{C}_{1}, \mathcal{C}_{2}, \mathcal{C}_{3}\right]=[-1,1,0]$ for the Weyl point $W_{1}$, and $\mathbf{C}=$ $[0,1,-1]$ for $W_{2}$. When both $W_{1}$ and $W_{2}$ are enclosed by $\mathbf{S}$, we have $\mathbf{C}=[-1,2,-1]$. In Fig. $4 \mathrm{a}$ in the main text, we plot the Berry curvature distribution of the first band around $W_{1}$. Figs. $6 \mathrm{a}$ and $6 \mathrm{~b}$ show the corresponding Berry curvatures for the other two bands, we see that the total flux for the second (third) band is quantized to 1 (0). Such non-trivial topology can also be characterized by the spin (vector and tensor) textures. For the first band, the spin tensor is rotated by $\pi$ on the loop $\mathcal{L}$ : $k_{x}=r_{S} \cos \theta, k_{y}=r_{S} \sin \theta, k_{z}=0$ with $\theta \in[0,2 \pi)$, and the spin vector crosses the center of the Bloch sphere once (as shown in Figs. 4c and $4 \mathrm{~d}$ in the main text), leading to the generalized solid angle $\gamma_{F}=0, \gamma_{T}=\pi$. Similarly, for the second band which is also non-trivial around $W_{1}$, the spin tensor is also rotated by $\pi$ on the loop $\mathcal{L}$ [see Fig. 6c], while the spin vector crosses the center of the Bloch sphere three times (see Fig. $4 \mathrm{~d}$ in the main text), leading to the generalized solid angle $\gamma_{F}=0, \gamma_{T}=\pi$. For the trivial third band around $W_{1}$, neither $\pi$-rotation for the spin tensor nor Bloch center crossing for the spin vector would exist [see Fig. 6d], leading to the generalized solid angle $\gamma_{F}=0, \gamma_{T}=0$. We may also consider a different loop $\mathcal{L}^{\prime}: k_{z}=r_{S} \cos \theta, k_{x}=r_{S} \sin \theta, k_{y}=0$ 
with $\theta \in[0,2 \pi)$ around $W_{1}$, and the spin textures are quite similar with the loop $\mathcal{L}$.

When both $W_{1}$ and $W_{2}$ are enclosed by the momentum surface $\mathbf{S}$, the Berry flux is quantized as $\mathbf{C}=[-1,2,-1]$ [as shown in Figs. 7a and 7c for the first two bands where $\mathbf{S}$ is a cylinder covering the whole Brillouin zone in $k_{z}$ ]. The spin vector distributions are shown in Figs. $7 \mathrm{~b}$ and $7 \mathrm{~d}$ for the first two bands. We see that there is a vortex at $k_{z}=0$ for the first band, and six vortices (three at $k_{z}=0$ and the other three at $k_{z}=\pi$ ) for the second band. While the Berry flux and spin distributions for the third band are similar with that for the first band, except that the vortex is located at $k_{z}=\pi$.

\section{Geometric representation of Berry flux}

Consider the parameter $\tau$-dependent Hamiltonian $H(\tau)$. For an arbitrary loop in the parameter space $\tau \in$ $\left[\tau_{\mathrm{i}}, \tau_{\mathrm{f}}\right]$ with the Hamiltonian satisfying $H\left(\tau_{\mathrm{i}}\right)=H\left(\tau_{\mathrm{f}}\right)$, the corresponding Berry phase of a given gapped eigenstate is

$$
\gamma=i \int d \tau\left\langle\Psi(\tau)\left|\partial_{\tau}\right| \Psi(\tau)\right\rangle+\gamma_{\mathrm{f}, \mathrm{i}},
$$

where $|\Psi(\tau)\rangle$, a smooth function of $\tau$, is the eigenstate of $H(\tau)$, and $\gamma_{\mathrm{f}, \mathrm{i}}$ is the gauge difference between two ends of the loop that is given by $\left|\Psi\left(\tau_{\mathrm{f}}\right)\right\rangle=e^{i \gamma_{\mathrm{f}, \mathrm{i}}}\left|\Psi\left(\tau_{\mathrm{i}}\right)\right\rangle$. We choose four parameters $F(\tau), \phi_{F}(\tau), \theta_{F}(\tau), \phi_{T}(\tau)$ to ensure a smooth wavefunction $|\Psi(\tau)\rangle=\left|\Psi\left[F(\tau), \phi_{F}(\tau), \theta_{F}(\tau), \phi_{T}(\tau)\right]\right\rangle$. Substitute Eq. (6) into Eq. (19), we obtain

$$
\gamma=\int\left[F d \phi_{T}+F \cos \left(\theta_{F}\right) d \phi_{F}\right]+\gamma_{\mathrm{f}, \mathrm{i}}
$$

with $\gamma_{\mathrm{f}, \mathrm{i}}=\left[\phi_{F}\left(\tau_{\mathrm{i}}\right)-\phi_{F}\left(\tau_{\mathrm{f}}\right)\right]+\left[\phi_{T}\left(\tau_{\mathrm{i}}\right)-\phi_{T}\left(\tau_{\mathrm{f}}\right)\right]$. We now define the generalized solid angle on the loop for the spin vector and tensor as $\gamma_{F}$ and $\gamma_{T}$, so that

$$
\begin{aligned}
\gamma_{F} & \equiv\left[\phi_{F}\left(\tau_{\mathrm{i}}\right)-\phi_{F}\left(\tau_{\mathrm{f}}\right)\right]+\int F \cos \left(\theta_{F}\right) d \phi_{F}, \\
\gamma_{T} & \equiv\left[\phi_{T}\left(\tau_{\mathrm{i}}\right)-\phi_{T}\left(\tau_{\mathrm{f}}\right)\right]+\int F d \phi_{T}, \\
\gamma & =\gamma_{F}+\gamma_{T} .
\end{aligned}
$$

From the definition, we see that $\gamma_{F}\left(\gamma_{T}\right)$ corresponds to the rotation of the spin vector (tensor). As an example, we consider the loop $\mathcal{L}$ in momentum space (by replacing the parameter $\tau$ with $\mathbf{k}$ ), and find that $\gamma_{F}=0, \gamma_{T}=\pi$. For a small enough loop, the Berry phase $\gamma$ gives the local Berry flux through the surface enclosed by the loop.

We want to emphasize that, to ensure a smooth wave function $|\Psi(\tau)\rangle, F(\tau), \phi_{F}(\tau), \theta_{F}(\tau), \phi_{T}(\tau)$ should also be a smooth function of $\tau$ except the points where $\langle\mathbf{F}\rangle$ crosses the $z$-axis on or inside the Bloch sphere, where
$\phi_{F}(\tau), \theta_{F}(\tau), \phi_{T}(\tau)$ may have jumps. We can simply remove these points in the integral that do not affect the final results.

\section{Majorana star representation of Berry flux}

An arbitrary spin-1 quantum state can be written as $|\Psi\rangle=\sum_{s} f_{s}|s\rangle$, and we will use $s=-1,0,1$ to represent the spin state $\downarrow, 0, \uparrow$ for convenience. We can rewrite the spin- 1 basis using the two-mode boson basis with $|s\rangle=\frac{\left(c^{\dagger}\right)^{1+s}\left(d^{\dagger}\right)^{1-s}}{(1+s) !(1-s) !}|\emptyset\rangle$, then the state can be factorized as $|\Psi\rangle=\frac{1}{\mathcal{N}} \prod_{j=1}^{2} \alpha_{j}^{\dagger}|\emptyset\rangle$, with $\mathcal{N}$ the normalization coefficient and $\alpha_{j}^{\dagger}=\cos \left(\theta_{j} / 2\right) c^{\dagger}+\sin \left(\theta_{j} / 2\right) e^{i \varphi_{j}} d^{\dagger}$. If we denote $c^{\dagger}|\emptyset\rangle$ and $d^{\dagger}|\emptyset\rangle$ as the spin-1/2 basis, then the above factorization will give out 2 pairs of parameters $\left(\theta_{j}, \varphi_{j}\right)$ which corresponds to 2 Majorana stars $\mathbf{m}_{j}=\left(\sin \theta_{j} \cos \varphi_{j}, \sin \theta_{j} \cos \varphi_{j}, \cos \theta_{j}\right)$ on the Bloch sphere. The parameters are determined by [55] $q_{j}=$ $\tan \left(\theta_{j} / 2\right) e^{i \varphi_{j}}$ with $q_{1}$ and $q_{2}$ the roots of the equation $\sum_{j=0}^{2} \frac{(-1)^{j} f_{1-j}}{\sqrt{j !(2-j) !}} q^{2-j}=0$. The Berry phase accumulated along a loop can be formulated as [55, 71]

$$
\begin{aligned}
\gamma= & i \int d \tau\left\langle\Psi(\tau)\left|\partial_{\tau}\right| \Psi(\tau)\right\rangle+\gamma_{\mathrm{f}, \mathrm{i}} \\
= & -\frac{1}{2} \oint \frac{\mathbf{m}_{1} \times \mathbf{m}_{2} \cdot\left(d \mathbf{m}_{1}-d \mathbf{m}_{2}\right)}{3+\mathbf{m}_{1} \cdot \mathbf{m}_{2}} \\
& -\sum_{j=1}^{2} \frac{1}{2} \oint\left(1-\cos \theta_{j}\right) d \varphi_{j}+\gamma_{\mathrm{f}, \mathrm{i}}
\end{aligned}
$$

The first term arises from the correlations between the two Majorana stars and the second term denotes the solid angles traced out by them. For the loop $\mathcal{L}$ in Fig. $4 \mathrm{e}, \gamma_{\mathrm{f}, \mathrm{i}}$ and the correlation term are both zero, and the Berry flux is determined solely by the solid angle traced out by the two Majorana stars.

* Corresponding author.

Email: chuanwei.zhang@utdallas.edu

[1] H. Weyl, Elektron und gravitation. I, Z. Phys. 56, 330 (1929).

[2] X.-G. Wan, A. M. Turner, A. Vishwanath, and S. Y. Savrasov, Topological semimetal and fermi-arc surface states in the electronic structure of pyrochlore iridates, Phys. Rev. B 83, 205101 (2011).

[3] A. M. Turner and A. Vishwanath. Beyond band insulators: Topology of semi-metals and interacting phases, arXiv:1301.0330.

[4] P. Hosur and X.-L. Qi. Recent developments in transport phenomena in Weyl semimetals, C. R. Phys 14, 857 (2013).

[5] Y. X. Zhao and Z. D. Wang, Disordered Weyl semimetals and their topological family, Phys. Rev. Lett. 114, 
206602 (2015).

[6] A. A. Zyuzin and A. A. Burkov, Topological response in Weyl semimetals and the chiral anomaly, Phys. Rev. B 86, 115133 (2012).

[7] Z. Wang and S.-C. Zhang, Chiral anomaly, charge density waves, and axion strings from Weyl semimetals, Phys. Rev. B 87, 161107 (2013).

[8] A. Martín-Ruiz, M. Cambiaso, and L. F. Urrutia, Electromagnetic fields induced by an electric charge near a Weyl semimetal, Phys. Rev. B 99, 155142 (2019).

[9] A. A. Burkov and L. Balents, Weyl semimetal in a topological insulator multilayer, Phys. Rev. Lett. 107, 127205 (2011).

[10] S.-M. Huang, S.-Y. Xu, I. Belopolski, C.-C. Lee, G.Q. Chang, B.-K. Wang, N. Alidoust, G. Bian, M. Neupane, C.-L. Zhang, S. Jia, A. Bansil, H. Lin, and M. Z. Hasan, A Weyl fermion semimetal with surface fermi arcs in the transition metal monopnictide taas class, Nat. Commun. 6, 7373 (2015).

[11] S.-Y. Xu, et al., Discovery of a Weyl fermion semimetal and topological fermi arcs, Science 349, 613 (2015).

[12] B. Q. Lv, et al., Experimental discovery of Weyl semimetal TaAs, Phys. Rev. X 5, 031013 (2015).

[13] S. A. Parameswaran, T. Grover, D. A. Abanin, D. A. Pesin, and A. Vishwanath, Probing the chiral anomaly with nonlocal transport in three-dimensional topological semimetals, Phys. Rev. X 4, 031035 (2014).

[14] A. A. Soluyanov, D. Gresch, Z.-J. Wang, Q.-S. Wu, M. Troyer, X. Dai, and B. A. Bernevig, Type-II Weyl semimetals, Nature 527, 495 (2015).

[15] Z.-J. ang, D. Gresch, A. A. Soluyanov, W.-W. Xie, S. Kushwaha, X. Dai, M. Troyer, R. J. Cava, and B. A. Bernevig, $\mathrm{MoTe}_{2}$ : A type-II Weyl topological metal, Phys. Rev. Lett. 117, 056805 (2015).

[16] T.-R. Chang, et al., Prediction of an arc-tunable Weyl fermion metallic state in $\mathrm{Mo}_{\mathrm{x}} \mathrm{W}_{1-\mathrm{x}} \mathrm{Te}_{2}$, Nat. Commun. 7, 10639 (2016).

[17] K. Deng, et al., Experimental observation of topological fermi arcs in type-II Weyl semimetal $\mathrm{MoTe}_{2}$, Nat. Phys. 12, 1105 (2016).

[18] L. Huang, T. M. McCormick, M. Ochi, Z. Zhao, M.T. Suzuki, R. Arita, Y. Wu, D. Mou, H. Cao, J. Yan, N. Trivedi, and A. Kaminski, Spectroscopic evidence for a type II Weyl semimetallic state in $\mathrm{MoTe}_{2}$, Nat. Mater. 15, 1155 (2016).

[19] Z. Lan, N. Goldman, A. Bermudez, W. Lu, and P. Öhberg, Dirac-Weyl fermions with arbitrary spin in two-dimensional optical superlattices, Phys. Rev. B 84, 165115 (2011).

[20] B. M. Anderson, G. Juzeliūnas, V. M. Galitski, and I. B. Spielman, Synthetic 3d spin-orbit coupling, Phys. Rev. Lett. 108, 235301 (2012).

[21] S. Ganeshan and S. Das Sarma. Constructing a Weyl semimetal by stacking one-dimensional topological phases, Phys. Rev. B 91, 125438 (2015).

[22] J.-H. Jiang, Tunable topological Weyl semimetal from simple-cubic lattices with staggered fluxes, Phys. Rev. A 85, 033640 (2012).

[23] T. Dubček, C. J. Kennedy, L. Lu, W. Ketterle, M. Soljačić, and H. Buljan, Weyl points in three-dimensional optical lattices: Synthetic magnetic monopoles in momentum space, Phys. Rev. Lett. 114, 225301 (2015).
[24] Y. Xu and L.-M. Duan, Type-II Weyl points in threedimensional cold-atom optical lattices, Phys. Rev. A 94, 053619 (2016).

[25] W.-Y. He, S. Zhang, and K. T. Law, Realization and detection of Weyl semimetals and the chiral anomaly in cold atomic systems, Phys. Rev. A 94, 013606 (2016).

[26] B.-Z. Wang, Y.-H. Lu, W. Sun, S. Chen, Y. Deng, and X.-J. Liu, Dirac-, rashba-, and Weyl-type spin-orbit couplings: Toward experimental realization in ultracold atoms, Phys. Rev. A 97, 011605 (2018).

[27] L. Lu, L. Fu, J. D. Joannopoulos, and M. Soljačić, Weyl points and line nodes in gyroid photonic crystals, Nat. Photon. 7, 294 (2013).

[28] W.-J. Chen, M. Xiao, and C. T. Chan, Photonic crystals possessing multiple Weyl points and the experimental observation of robust surface states, Nat. Commun. 7, 13038 (2016).

[29] L. Lu, Z. Wang, D. Ye, L. Ran, L. Fu, J. D. Joannopoulos, and M. Soljačić, Experimental observation of Weyl points, Science 349, 622 (2015).

[30] M. Xiao, Q. Lin, and S. Fan, Hyperbolic Weyl point in reciprocal chiral metamaterials, Phys. Rev. Lett. 117, 057401 (2016).

[31] Q. Lin, M. Xiao, L. Yuan, and S. Fan, Photonic Weyl point in a two-dimensional resonator lattice with a synthetic frequency dimension, Nat. Commun. 7, 13731 (2016).

[32] B.-Y. Sun, X.-W. Luo, M. Gong, G.-C. Guo, and Z.W. Zhou, Weyl semimetal phases and implementation in degenerate optical cavities, Phys. Rev. A 96, 013857 (2017).

[33] M. Xiao, W.-J. Chen, W.-Y. He, and C. T. Chan, Synthetic gauge flux and Weyl points in acoustic systems, Nat. Phys. 11, 920 (2015).

[34] Y.-J. Lin, K. Jiménez-García, and I. B. Spielman, Spinorbit-coupled Bose-Einstein condensates, Nature (London) 471, 83, (2011).

[35] J.-Y. Zhang, S.-C. Ji, Z. Chen, L. Zhang, Z.-D. Du, B. Yan, G.-S. Pan, B. Zhao, Y.-J. Deng, H. Zhai, S. Chen, and J.-W. Pan, Collective dipole oscillations of a spinorbit coupled Bose-Einstein condensate, Phys. Rev. Lett. 109, 115301, (2012).

[36] C. Qu, C. Hamner, M. Gong, C. Zhang, and P. Engels, Observation of zitterbewegung in a spin-orbit-coupled Bose-Einstein condensate, Phys. Rev. A 88, 021604, (2013).

[37] S.-C. Ji, J.-Y. Zhang, L. Zhang, Z.-D. Du, W. Zheng, Y.J. Deng, H. Zhai, S. Chen, and J.-W. Pan, Experimental determination of the finite-temperature phase diagram of a spin-orbit coupled Bose gas, Nat. Phys. 10, 314 (2014).

[38] A. Olson, S. Wang, R. Niffenegger, C. Li, C. Greene, and Y. Chen, Tunable landau-zener transitions in a spinorbit-coupled Bose-Einstein condensate, Phys. Rev. A 90, 013616, (2014).

[39] P. Wang, Z. Yu, Z. Fu, J. Miao, L. Huang, S. Chai, H. Zhai, and J. Zhang, Spin-orbit coupled degenerate fermi gases, Phys. Rev. Lett. 109, 095301, (2012).

[40] L. Cheuk, A. Sommer, Z. Hadzibabic, T. Yefsah, W. Bakr, and M. Zwierlein, Spin-injection spectroscopy of a spin-orbit coupled fermi gas, Phys. Rev. Lett. 109, 095302 (2012).

[41] Z. Meng, L. Huang, P. Peng, D. Li, L. Chen, Y. Xu, C. Zhang, P. Wang, and J. Zhang, Experimental observation of a topological band gap opening in ultracold Fermi 
gases with two-dimensional spin-orbit coupling, Phys. Rev. Lett. 117, 235304 (2016).

[42] L. Huang, Z. Meng, P. Wang, P. Peng, S. Zhang, L. Chen, D. Li, Q. Zhou, and J. Zhang, Experimental realization of two-dimensional synthetic spin-orbit coupling in ultracold fermi gases, Nat. Phys. 12, 540 (2016).

[43] Z. Wu, L. Zhang, W. Sun, X. Xu, B. Wang, S. Ji, Y. Deng, S. Chen, X.-J. Liu, and J. Pan, Realization of two-dimensional spin-orbit coupling for Bose-Einstein condensates, Science 354, 83 (2016).

[44] D. Campbell, R. Price, A. Putra, A. Valdés-Curiel, D. Trypogeorgos, and I. B. Spielman, Magnetic phases of spin-1 spin-orbit-coupled Bose gases, Nat. Commun. 7, 10897 (2016).

[45] X. Luo, L. Wu, J. Chen, Q. Guan, K. Gao, Z.-F. Xu, L. You and R. Wang, Tunable atomic spin-orbit coupling synthesized with a modulating gradient magnetic field, Sci. Rep. 6, 18983 (2016).

[46] J. Dalibard, F. Gerbier, G. Juzeliūnas, and P. Öhberg, Colloquium: Artificial gauge potentials for neutral atoms, Rev. Mod. Phys. 83, 1523 (2011).

[47] N. Goldman, G. Juzeliūnas, P. Öhberg, and I. B. Spielman, Light-induced gauge fields for ultracold atoms, Rep. Prog. Phys. 77, 126401 (2014).

[48] Y. Xu, F. Zhang, and C. Zhang, Structured Weyl points in spin-orbit coupled fermionic superfluids, Phys. Rev. Lett. 115, 265304 (2015).

[49] M. Gong, S. Tewari, and C. Zhang, BCS-BEC crossover and topological phase transition in $3 \mathrm{~d}$ spin-orbit coupled degenerate fermi gases, Phys. Rev. Lett. 107, 195303 (2011).

[50] Y. Xu, R.-L. Chu, and C. Zhang, Anisotropic Weyl fermions from the quasiparticle excitation spectrum of a 3d fulde-ferrell superfluid, Phys. Rev. Lett. 112, 136402 (2014).

[51] X. Zhou, X.-W. Luo, G. Chen, S. Jia, and C. Zhang, Rashba and Weyl spin-orbit coupling in an optical lattice clock, Phys. Rev. A 100, 063630 (2019).

[52] H. M. Bharath, Non-abelian geometric phases carried by the spin fluctuation tensor, J. Math. Phys. 59, 062105 (2018).

[53] H. M. Bharath, M. Boguslawski, M. Barrios, L. Xin, and M. S. Chapman, Exploring Non-Abelian Geometric Phases in Spin-1 Ultracold Atoms, Phys. Rev. Lett. 123, 173202 (2019).

[54] X. Zhou, X.-W. Luo, G. Chen, S. Jia, and C. Zhang, Quantum spiral spin-tensor magnetism, Phys. Rev. B 101, 140412 (2020).

[55] H. D. Liu and L. B. Fu, Representation of berry phase by the trajectories of majorana stars, Phys. Rev. Lett. 113, 240403, (2014).

[56] L. F. Livi, G. Cappellini, M. Diem, L. Franchi, C. Clivati, M. Frittelli, F. Levi, D. Calonico, J. Catani, M. Inguscio, and L. Fallani, Synthetic dimensions and spin-orbit coupling with an optical clock transition, Phys. Rev. Lett. 117, 220401, (2016).

[57] M. Mancini, G. Pagano, G. Cappellini, L. Livi, M. Rider, J. Catani, C. Sias, P. Zoller, M. Inguscio, M. Dalmonte, and L. Fallani, Observation of chiral edge states with neutral fermions in synthetic hall ribbons, Science 349, 1510 (2015).
[58] S. Kolkowitz, S. L. Bromley, T. Bothwell, M. L. Wall, G. E. Marti, A. P. Koller, X. Zhang, A. M. Rey, and J. Ye, Spin-orbit-coupled fermions in an optical lattice clock, Nature 542, 66 (2017).

[59] S. L. Bromley, S. Kolkowitz, T. Bothwell, D. Kedar, A. Safavi-Naini, M. L. Wall, C. Salomon, A. M. Rey, and J. Ye, Dynamics of interacting fermions under spinorbit coupling in an optical lattice clock, Nat. Phys. 14, 399 (2018).

[60] O. Pfister, J. S. Wells, L. Hollberg, L. Zink, D. A. Van Baak, M. D. Levenson, and W. R. Bosenberg, Continuous-wave frequency tripling and quadrupling by simultaneous three-wave mixings in periodically poled crystals: application to a two-step 1.19-10.71- $\mu \mathrm{m}$ frequency bridge, Opt. Lett. 22, 1211 (1997).

[61] A. B. Fedotov, S. M. Gladkov, N. I. Koroteev, and A. M. Zheltikov, Highly efficient frequency tripling of laser radiation in a low-temperature laser-produced gaseous plasma, J. Opt. Soc. Am. B 8, 363 (1991).

[62] M. Lohse, C. Schweizer, O. Zilberberg, M. Aidelsburger, and I. Bloch, A thouless quantum pump with ultracold bosonic atoms in an optical superlattice, Nat. Phys 12, 350 (2016).

[63] M. Aidelsburger, M. Lohse, C. Schweizer, M. Atala, J. T. Barreiro, S. Nascimbene, N. R. Cooper, I. Bloch, and N. Goldman, Measuring the chern number of hofstadter bands with ultracold bosonic atoms, Nat. Phys. 11, 162 (2015).

[64] J.-R. Li, W. Huang, B. Shteynas, S. Burchesky, F. Çă̆rı Top, E. Su, J. Lee, A. O. Jamison, and W. Ketterle, Spinorbit coupling and spin textures in optical superlattices, Phys. Rev. Lett. 117, 185301, (2016).

[65] J.-R. Li, J. Lee, W. Huang, S. Burchesky, B. Shteynas, F. Çă̆rı Top, A. O. Jamison, and W. Ketterle, A stripe phase with supersolid properties in spin-orbit-coupled bose-einstein condensates, Nature 543, 91 (2017).

[66] J. Zhang, H. Hu, X.-J. Liu, and H. Pu, Fermi gases with synthetic spin-orbit coupling, Annu. Rev. Cold At. Mol. 281 (2014).

[67] M. L. Wall, A. P. Koller, S. Li, X. Zhang, N. R. Cooper, J. Ye, and A. M. Rey, Synthetic spin-orbit coupling in an optical lattice clock, Phys. Rev. Lett. 116, 035301, (2016).

[68] B. Bradlyn, J. Cano, Z. Wang, M. G. Vergniory, C. Felser, R. J. Cava, and B. A. Bernevig, Beyond Dirac and Weyl fermions: Unconventional quasiparticles in conventional crystals, Science 353, aaf5037 (2016).

[69] B. Q. Lv, Z.-L. Feng, Q.-N. Xu, X. Gao, J.-Z. Ma, L.-Y. Kong, P. Richard, Y.-B. Huang, V. N. Strocov, C. Fang, H.-M. Weng, Y.-G. Shi, T. Qian, and H. Ding Observation of three-component fermions in the topological semimetal molybdenum phosphide, Nature 546, 627 (2017).

[70] H. Hu, J. Hou, F. Zhang, and C. Zhang, Topological Triply Degenerate Points Induced by Spin-TensorMomentum Couplings, Phys. Rev. Lett. 120, 240401 (2018).

[71] H. Hu and C. Zhang, Spin-1 topological monopoles in the parameter space of ultracold atoms, Phys. Rev. A 98, 013627 (2018). 